\title{
Erratum to: Development of Vascular Tissue and Stress Inducible Hybrid-Synthetic Promoters Through Dof-1 Motifs Rearrangement
}

\author{
Rajiv Ranjan • Nrisingha Dey
}

Published online: 7 December 2012

(c) Springer Science+Business Media New York 2012

\section{Erratum to: Cell Biochem Biophys (2012) 63:235-245 DOI 10.1007/s12013-012-9359-9}

Unfortunately the legends of Fig. 6 and the Fig. 8d were furnished incorrectly in the original publication of the article by mistake. These errors are corrected in this erratum as follows:

1. Corrected legend of Fig. 6:

CLSM assay. a Superimposed (bright field and fluorescent) images of transverse section of tobacco petiole expressing GUS in vascular tissue under the control of CaMV35S, EFCFS, EFCFS-HS-1, EFCFS-HS-2 and EFCFS-HS-3 promoter constructs. b Fluorescence images of transverse section of transgenic tobacco petiole expressing GUS in vascular tissue under the control of CaMV35S, EFCFS, EFCFS-HS-1, EFCFSHS-2 and EFCFS-HS-3 promoter constructs. c Bright field confocal images of transverse section of transgenic tobacco petiole expressing GUS in vascular tissue under the control of CaMV35S, EFCFS, EFCFSHS-1, EFCFS-HS-2 and EFCFS-HS-3 promoter constructs

2. Corrected Fig. 8d is presented below.

The online version of the original article can be found under doi:10.1007/s12013-012-9359-9.

\section{R. Ranjan · N. Dey $(\bowtie)$}

Division of Gene Function and Regulation, Institute of Life Sciences, Department of Biotechnology, Govt. of India, Nalco Square, Chandrasekherpur, Bhubaneswar 751023 , Orissa, India e-mail: dey@ils.res.in; nrisinghad@yahoo.com

R. Ranjan

e-mail: rajivranjanbt@gmail.com

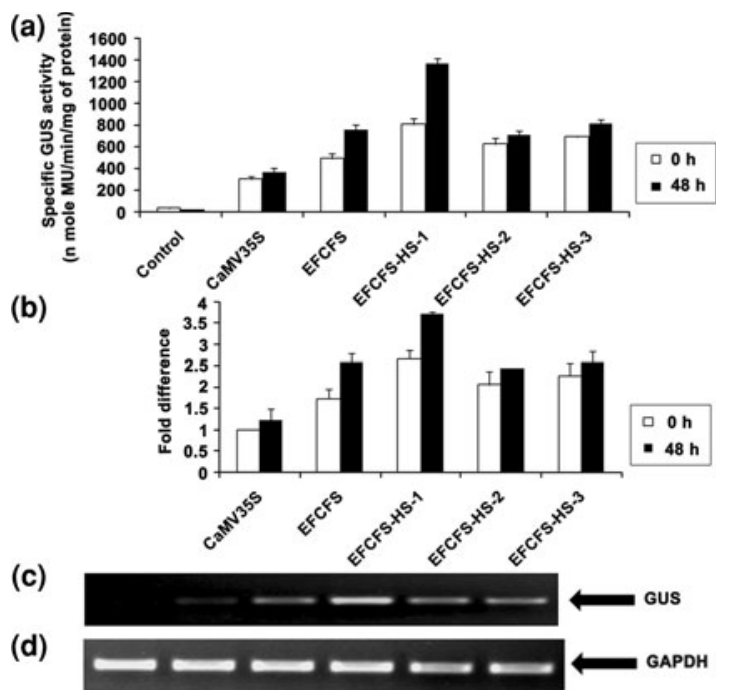

Fig. 8 JA induction. a Tobacco whole seedlings (21 days old) were treated with JA as described in 'Method' section. The GUS expression activities from control seedlings (transformed with no GUS), CaMV35S, EFCFS, EFCFS-HS-1, EFCFS-HS-2 and EFCFSHS-3 promoter constructs were monitored at 0 and $48 \mathrm{~h}$ of posttreatment. The data were presented as mean of three-independent experiments for each construct. Statistical analysis showed a $P$ value of $<0.003$, indicating highly significant. b Real-time PCR-based relative of GUS transcript accumulation (uidA-mRNA) in whole seedlings expressing CaMV35S, EFCFS, EFCFS-HS-1, EFCFS-HS-2 and EFCFS-HS-3 promoter constructs, 0 and $48 \mathrm{~h}$ post-treatment with JA. The data were presented as an average fold differences of $G U S$ transcript of two-independent experiments. Each bar represents fold difference in transcript level of the GUS gene in transgenic plants in comparison to that obtained in transgenic plants expressing CaMV35S (taken as 1) promoter. $\mathbf{c}$ Electrophoresis of RT-dependent PCR amplifications of GUS transcripts from total RNA (DNaseI treated) obtained from transgenic plant expressing CaMV35S, EFCFS, EFCFS-HS-1, EFCFS-HS-2 and EFCFS-HS-3 constructs. d Electrophoresis of RT-dependent PCR amplifications of GAPDH transcripts from total RNA (DNaseI treated) from transgenic plant expressing CaMV35S, EFCFS, EFCFS-HS-1, EFCFS-HS-2 and EFCFS-HS-3 promoter constructs 